\section{[gw22-e0015] EFFECTS OF TONGXINLUO CAPSULE ON HOMOCYSTEINE-INDUCED ENDOTHELIAL DYSFUNCION AND INFLAMMARTOY RESPONSE}

Zhou Bin' ${ }^{1}$ Liu Yong', Hao Baoshun', Liu Dinghui', Wang Ming', Wu Lin', Chen Lin', Liu Jinlai', Wu Weikang ${ }^{1}$ Department Of Cardiology, Third Affiliated Hospital, Sun YatSen University; ${ }^{2}$ Institute for Integrated Traditional Chinese and Western Medicine, Third Affiliated Hospital, Sun Yat-Sen University

\subsection{6/heartjnl-2011-300867.96}

Objective To investigate the effects of Tongxinluo (TXL) on Homocysteine (HCY)-induced endothelial dysfunction and inflammatory response.

Methods Isolation, culture and identification of human umbilical vein endothelial cells (HUVECs): With the informed consent of puerperants, the normal fetal umbilical cords were obtained through uterine-incision delivery in Third Affiliated Hospital, Sun Yat-sun University. HUVECs were isolated by Percoll density gradient centrifugation from fetal umbilical cords with digestion of collagenase type I perfusion, and then suspended in Medium 199 cultured in 0.05 volume fraction of $\mathrm{CO}_{2}$ incubator at $37^{\circ} \mathrm{C}$. After the HUVECs were identified by flow cytometry with the cell marker CD34, The second or third passage was used for study. MTT assay was used to detect cell viability: HUVECs during the logarithmic phase were inoculated in 96-well plates with Medium 199 in 0.05 volume fraction of $\mathrm{CO}_{2}$ incubator at $37^{\circ} \mathrm{C}$ for $48 \mathrm{~h}$, at $80 \%$ confluence, incubated in serum-free medium, and divided into two groups, each group including eight parallel wells, blank control group was untreated, the latter cultured and added with HCY of the concentrations of $0.1,0.25,0.5$, and $1.0 \mathrm{mmol} / \mathrm{l}$, respectively, or HCY $0.5 \mathrm{mmol} / \mathrm{l}+\mathrm{TXL}$ of the concentrations of $0.1,0.5,1.0$, and $2.0 \mathrm{mg} / \mathrm{ml}$. After the cells were cultured for $24 \mathrm{~h}, 5 \mathrm{~g} / \mathrm{l}$ MTT solution $(20 \mu \mathrm{l})$ was added, then dimethyl sulfoxide was used for vibration and dissolvement. Optical density of each hole was detected by using enzyme-linked immunosorbent assay to analyse different concentrations of HCY and TXL on the proliferation of human mammary stems cells. Western blot was used to examine the protein expression of the cell inflammatory factors, tumour necrosis factor (TNF) $\alpha$, interleukin (IL)- 6 , macrophage chemoattractant protein (MCP)-1, and intercellular adhesion molecule (ICAM)-1, ELISA was used to detect the contents of the cell inflammatory factors.

Results HCY of different doses inhibited the viability of HUVECs dose-dependently, the inhibition rates of HCY were $0.099 \pm 0.079,0.239 \pm 0.046,0.637 \pm 0.007$, and $0.553 \pm 0.029$, respectively (all $\mathrm{p}<0.01$ ); the growth rates of HCY-induced HUVECs treatment by TXL of the concentrations of 0.1 , $0.5,1.0$, and $2.0 \mathrm{mg} / \mathrm{ml}$ were $0.682 \pm 0.312,0.710 \pm 0.040$, $0.855 \pm 0.130,0.784 \pm 0.031$, respectively, higher than that of the control group (all $\mathrm{p}<0.01$ ). Western blotting and ELISA showed that homocysteine significantly increased the expression of TNF $\alpha$, IL- 6, MCP-1 and ICAM-1, which are $0.71 \pm 0.15$, $0.89 \pm 0.32,0.80 \pm 0.27,0.54 \pm 012$, respectively, higher than that of 0 hour (all $p<0.01$ ). However, the expression levels of TNF $\alpha$, IL-6, MCP-1 and ICAM-1 of the $0.50 \mathrm{mmol} / 1 \mathrm{HCY}$-induced HUVECs treatment by TXL $(1 \mathrm{mg} / \mathrm{ml})$ were $0.24 \pm 0.04$, $0.15 \pm 0.03,0.13 \pm 0.04,0.22 \pm 0.06$, respectively, not significantly different from those at the of 0 hour (all p>0.01).

Conclusion Tongxinluo inhibits homocysteine-induced endothelial impairment and inflammatory response, the definite mechanism of which requires further research. 